\title{
BAYESIAN SINGLE-EPOCH PHOTOMETRIC CLASSIFICATION OF SUPERNOVAE
}

\author{
Dovi Poznanski and Dan MaOz \\ School of Physics and Astronomy, Tel-Aviv University, Tel-Aviv 69978, Israel; dovip@wise.tau.ac.il, dani@wise.tau.ac.il \\ AND \\ Avishay GaL-Yam ${ }^{1}$ \\ Astronomy Department, California Institute of Technology, MS 105-24, Pasadena, CA 91125, USA; \\ avishay@astro.caltech.edu \\ Received 2006 October 4; accepted 2007 June 17
}

\begin{abstract}
Ongoing supernova (SN) surveys find hundreds of candidates that require confirmation for their various uses. Traditional classification based on follow-up spectroscopy of all candidates is virtually impossible for these large samples. The use of Type Ia SNe as standard candles is at an evolved stage that requires pure, uncontaminated samples. However, other SN survey applications, such as measuring cosmic SN rates, could benefit from a classification of $\mathrm{SNe}$ on a statistical basis, rather than case by case. With this objective in mind, we have developed the SN-ABC, an automatic Bayesian classifying algorithm for supernovae. We rely solely on single-epoch multiband photometry and host-galaxy (photometric) redshift information to sort SN candidates into the two major types, Ia and corecollapse supernovae. We test the SN-ABC performance on published samples of SNe from the Supernova Legacy Survey (SNLS) and GOODS projects that have both broadband photometry and spectroscopic classification (so the true type is known). The SN-ABC correctly classifies up to $97 \%$ (85\%) of the Type Ia (II-P) SNe in SNLS, and similar fractions of the GOODS SNe, depending on photometric redshift quality. Using simulations with large artificial samples, we find similarly high success fractions for Types Ia and II-P, and reasonable $(\sim 75 \%)$ success rates in classifying Type Ibc SNe as core-collapse. Type IIn SNe, however, are often misclassified as Type Ia. In deep surveys, SNe Ia are best classified at redshifts $z \gtrsim 0.6$ or when near maximum. Core-collapse SNe (other than Type IIn) are best recognized several weeks after maximum, or at $z \lesssim 0.6$. Assuming the SNe are young, as would be the case for rolling surveys, the success fractions improve by a degree dependent on the type and redshift. The fractional contamination of a single-epoch photometrically selected sample of SNe Ia by core-collapse SNe varies between less than $10 \%$ and as much as $30 \%$, depending on the intrinsic fraction and redshift distribution of the corecollapse SNe in a given survey. The SN-ABC also allows the rejection of SN "impostors" such as active galactic nuclei (AGNs), with half of the AGNs we simulate rejected by the algorithm. Our algorithm also supplies a good measure of the quality of the classification, which is valuable for error estimation.
\end{abstract}

Key word: supernovae: general

Online material: color figures

\section{INTRODUCTION}

Supernovae ( $\mathrm{SNe}$ ) are usually subdivided into different types, based on spectroscopic or photometric criteria (e.g., Filippenko 1997). The primary physical distinction among the common supernova $(\mathrm{SN})$ types separates core-collapse $\mathrm{SNe}(\mathrm{CC}-\mathrm{SNe})$, which originate from the collapse of a massive star (e.g., Woosley \& Janka 2005), from Type Ia SNe (SNe Ia), for which there are competing scenarios, generally involving a thermonuclear runaway in a degenerate system (e.g., Hillebrandt \& Niemeyer 2000). Recent technological advances in wide-field imaging and in automation capabilities, combined with a growing interest in transient phenomena, have led to numerous surveys that find hundreds of SNe. These surveys search for SNe nearby (Filippenko et al. 2001; Wood-Vasey et al. 2004), at intermediate redshifts (Dilday et al. 2005; Cappellaro et al. 2005), and at high redshifts (Poznanski et al. 2007; Astier et al. 2006; Riess et al. 2004a; Sollerman et al. 2006). While most ongoing SN searches focus on the use of SNe Ia as standard candles for cosmological applications (Riess et al. 2004a; Astier et al. 2006), large SN samples can be used also to study the statistical properties of the $\mathrm{SNe}$

\footnotetext{
${ }^{1}$ Hubble Fellow.
}

themselves, and to measure their rates as a function of cosmic time (e.g., Maoz \& Gal-Yam 2004; Dahlen et al. 2004). Because the lifetimes of massive stars are short compared to cosmological timescales, the rate of CC-SNe is expected to follow the star formation history, and thus could provide an independent measure of this function (e.g., Dahlén \& Fransson 1999). The rate of SNe Ia is linked to star formation through a more complicated evolutionary process that depends on the nature of the progenitor systems. Measurement of the SN Ia rate may therefore constrain possible progenitor models (e.g., Madau et al. 1998; Gal-Yam \& Maoz 2004; Strolger et al. 2004; Scannapieco \& Bildsten 2005; Mannucci et al. 2005, 2006; Sullivan et al. 2006b; Förster et al. 2006).

Future projects such as Pan-STARRS (Kaiser et al. 2005), the Large Synoptic Survey Telescope (Stubbs et al. 2004), and the Supernova/Acceleration Probe (Aldering 2005), promise to increase the yield of SNe by orders of magnitude, especially at intermediate and high redshifts. All of the SN studies above require large, well-defined samples of $\mathrm{SNe}$ with determined types and redshifts. The common methodology of attempting to obtain spectroscopic follow-up of every SN candidate, in order to determine its type, its redshift (based on the host spectrum or from the $\mathrm{SN}$ spectrum itself), and possibly other characteristics, 
is unsuitable for this plethora of SNe, due to their faintness, sheer numbers, or both. For this reason, current surveys usually focus on subsets of the SN candidates for which spectroscopic observations can be obtained. Ongoing surveys already employ some photometric criteria for prioritizing follow-up, but usually not for definitive classification, as we discuss below.

In Poznanski et al. (2002) and Gal-Yam et al. (2004) we showed, using color-color diagrams, that given a known redshift, different types of SNe can often be distinguished based on colors alone. Our method and Web tool ${ }^{2}$ have been used, among others, by Rajala et al. (2005) to classify nearby SNe, by Gal-Yam et al. (2005) to choose objects for follow-up prior to spectroscopy, and by Strolger et al. (2004) for high-redshift CC-SN identification. The approach has been developed further by several studies. Riess et al. (2004b) showed that at high redshift, it might be even easier to distinguish, near maximum light, between SNe Ia and $\mathrm{CC}-\mathrm{SNe}$ by virtue of the difference in the rest-frame UV emission between these two classes of objects. Johnson \& Crotts (2006) investigated further the color-magnitude-based classification of intermediate-redshift SNe with different filter sets both with and without redshift information. Sullivan et al. (2006a) introduced the use of automatic photometric classification as part of the reduction pipeline of the Supernova Legacy Survey (SNLS; Astier et al. 2006), using two to three early light-curve points. The objective was mainly to recognize SNe Ia, with a minimum of false positives and selection biases, and thus to prioritize targets for follow-up spectroscopy. Barris \& Tonry (2006) fit complete light curves and applied various quality cuts in order to recognize SNe Ia among the various transients they found in their survey (see also Barris \& Tonry 2004).

In this paper we present an algorithm, the SN Automatic Bayesian Classifier (SN-ABC), intended for automatic classification of SNe using single-epoch photometry and whatever redshift information is available. The main novelties of our algorithm lie in the probabilistic and Bayesian methodologies. Our approach is probabilistic in the sense that we do not expect to succeed with the classification of every specific object but to correctly classify most objects, while minimizing biases in the output sample, this by assigning each object its most likely type. The flexibility of the Bayesian framework allows us to do so by analyzing SNe from different surveys, with different depths and types of redshift information, incorporating fully all the available information on each object, and propagating correctly the unknowns. As a proof of concept, we focus on single-epoch photometry in three bands, but the method can be straightforwardly implemented with multiply sampled light curves with other photometric setups. As we show below, SNe can be classified quite reliably even with meager input data, and therefore, numerous $\mathrm{SNe}$ can be found and analyzed at a relatively low cost in telescope time.

We test our method on light curves, recently released by the SNLS, of 71 SNe Ia and five "plateau-type" (II-P) SNe (Astier et al. 2006; Nugent et al. 2006), and on the sample of $42 \mathrm{SNe}$ found during the GOODS campaign (Dahlen et al. 2004). A third sample of simulated SN data is used to investigate potential biases and the efficiency of the method as a function of SN and sample parameters. In a separate paper (Poznanski et al. 2007) we use our method to classify a sample of SNe we have found in the Subaru Deep Field (SDF; Kashikawa et al. 2004), with the objective of measuring the rates of SNe Ia and CC SNe at high redshift based on this data set.

Shortly prior to submission of this paper, Kuznetsova \& Connolly (2007) presented a Bayesian classification algorithm

\footnotetext{
${ }^{2}$ See http://wise-obs.tau.ac.il/ dovip/typing/.
}

and its application to SNLS and GOODS data. Although there are some similarities between their approach and ours, there are also some major differences. First, while they use complete light curves, and discuss the importance of such data, we focus on single-epoch observations, in which information is scarce and classification is less obvious. Second, they treat the redshift of the SN as a known quantity without any ambiguity. While this is true for most of the $\mathrm{SNe}$ in the training samples they (and we) use, this is certainly not the case for existing and future SN samples, where methods of photometric classification apply. In our work, while we focus on the case where photometric redshifts for the host galaxies of the $\mathrm{SNe}$ are available, we treat the general case, where each object can have any possible redshift estimation, from precise (spectroscopic) to totally unavailable. In each case the uncertainties in the redshift measurement are taken into account. Finally, Kuznetsova \& Connolly (2007) estimate the probabilities after marginalizing over three extinction scenarios (no extinction, $R_{V}=3.1$ with $A_{V}=0.4$, and $R_{V}=2.1$ with $A_{V}=0.4$ ). As explained in $\S 2.2$, we assume a range of extinction models with $R_{V}=3.1$, and $A_{V}$ between 0 and 1 .

\section{METHOD}

Our general approach for SN classification relies on a Bayesian template-fitting technique. Given a "prior" on the redshift of a $\mathrm{SN}$ candidate, we fit a set of template spectral energy distributions (SEDs) to the observed photometry of the SN. We then maximize the likelihood in order to find the characteristics of the $\mathrm{SN}$, derive the evidence for the object being either a SN Ia or a $\mathrm{CC}-\mathrm{SN}$, and find its posterior redshift distribution function. We describe below each step in detail.

\subsection{SN Photometry and Prior (Redshift) Information}

We assume the availability of single-epoch multiband photometry of the candidate SNe. As we show below, this is probably the minimum amount of data that can produce scientifically meaningful results, and is also the kind of information we have for the SDF (Poznanski et al. 2007). However, this method can be easily applied to cases where more information is available, including complete multiband light curves, with a guaranteed improvement in the results.

We further assume the existence of a prior redshift probability distribution function (z-pdf) for the SNe. Such a prior distribution would come naturally from the photometric redshifts of the host galaxies, but in principle could be from any source. Photometric redshifts are now being produced by every major extragalactic survey, and for well-studied fields there may even be an available spectrum of the host galaxy, or one can be obtained later when the SN has faded. (In the Bayesian formulation, a spectroscopic redshift corresponds to a sharply peaked prior $z$-pdf.) In cases where there is no redshift information (e.g., because the host is too faint) one can assume a flat, or some other physically motivated, $z$-pdf.

The Bayesian approach permits a careful treatment of the unknowns, which can be propagated fully from one step of the analysis to the next. Even when the redshift is derived from spectroscopy, it can have uncertainties which are hard to propagate further in any frequentist approach. Here we can treat selfconsistently redshift information of any given quality, whether a narrow, peaked $z$-pdf from a spectral redshift, a broad or oddly shaped redshift distribution, including redshift limits, or even a nonexistent photometric redshift, all jointly analyzed. Furthermore, when interested in the redshift distribution of the $\mathrm{SNe}$, one can use the full posterior redshift distribution for each object, 
rather than a single value derived from its peak, and thus propagate further the complete information. Finally, in "rolling searches," where all SNe are found when rising or near peak, one can similarly include a prior on the age of the $\mathrm{SN}$, and thus improve the classification.

\subsection{Model}

We use SN templates from the updated version of the $\mathrm{SN}$ SEDs of Nugent et al. (2002). ${ }^{3}$ These include sets of redshift $z=0$ spectra of SNe of different types and at different epochs, covering the near-IR to the rest-frame UV. After experimenting with various simulations and applications to real data, most of which are presented in $\S \S 3$ and 4, we find that the best classification, and the fewest subsequent biases, in type and redshift distributions are achieved when only the templates for Types II-P and normal (stretch $s=1$ ) Ia are used in the fits. As we show in $\S 4$, we find that, with the exception of Type IIn SNe (see discussion in $\S 4$ ), most CC-SNe are more similar on average to Type II-P than to Type Ia SNe. Since our main objective will be an empirical division between the two main physical classes of $\mathrm{SNe}$, Ia and $\mathrm{CC}$, we retain only these two types, using the II-P templates as proxies for the whole CC SN class. In general template-fitting algorithms, the use of many templates, including relatively rare objects, tends to damage the fit of the more common objects (e.g., Benítez 2000). In our case, adding templates for other CC-SNe, while somehow improving the classification of these types of SNe, causes many SNe Ia to be wrongly classified and thus lowers the overall success rate of our algorithm. We emphasize that while we use those two specific template families to measure whether a given object is a SN Ia or a CC-SN, we are concerned with the classification of all types of SNe. Our working assumption (which is largely confirmed by the results we present in the following sections) is that most Type Ia SNe have colors and magnitudes more similar to those of a normal SN Ia than to those of a Type II-P SN, and conversely, that most $\mathrm{CC}-\mathrm{SNe}$, including the various subtypes, are more similar to a II-P type than to a SN Ia.

Our adopted templates span a range in $\mathrm{SN}$ age, $t$, from about 2 weeks prior to maximum $B$-band light to $2(3)$ months past maximum for the Ia (CC) SNe. Using synthetic photometry, we have scaled the template spectra to reach at maximum light the $B$-band absolute magnitudes in Table 1 of Dahlen et al. (2004), which are based on the works of Richardson et al. (2002), Li et al. (2001), and Tonry et al. (2003). We consider two types of intrinsic dispersion in SN properties: one in the absolute magnitude, which we take from Dahlen et al. (2004), and one in color, of $0.1 \mathrm{mag}$ (for whatever color is being considered, e.g., $r-i$ and $i-z$ ). The latter is consistent with what has been measured for SNe Ia by Nobili et al. (2003), Jha et al. (2006), and S. Ellis et al. (2007, in preparation), while for CC-SNe we are not aware of the existence of similar published data (although see Elmhamdi et al. 2003).

We apply to the template spectra a grid of redshifts (from 0 to 2 ) and extinctions $\left(A_{V}=0-1 \mathrm{mag}\right)$ at the $\mathrm{SN}$ redshift using the Cardelli et al. (1989) extinction law, with $R_{V}=3$.1. Luminosity distances are calculated using the currently favored cosmology ( $h=0.7, \Omega_{\Lambda}=0.7, \Omega_{m}=0.3$ ). Finally, we apply a range of observer-frame Galactic extinctions to the spectra (from zero extinction to $A_{V}=0.1$, the maximal value for the $\mathrm{SNe}$ in the GOODS and SNLS surveys), again using the reddening law of Cardelli et al. (1989).

\footnotetext{
${ }^{3}$ Available at http://supernova.lbl.gov/ nugent/nugent_templates.html.
}

We calculate synthetic magnitudes from the SEDs, based on the bandpasses used in the particular survey being analyzed. These bandpasses are the products of the filter transmission curves, detector quantum efficiency curves, and (for ground-based data) atmospheric transmission. We thus obtain a five-dimensional data cube of model magnitudes for each type of SN as a function of redshift, age, band, host extinction, and Galactic extinction, from which we select for each object a four-dimensional cube according to the expected Galactic extinction from the maps of Schlegel et al. (1998). We perform all of our analysis using Vega-based magnitudes.

\subsection{Fitting}

For every SN we classify, we calculate two three-dimensional posterior likelihood matrices, one assuming it is a SN Ia, and one assuming it is a CC-SN:

$$
L_{\text {type }}\left(z, A_{V}, t\right)=P(z) \exp \left\{-\sum_{i}\left[\frac{m_{\bmod , i}\left(z, A_{V}, t\right)-m_{i}}{d m_{i}}\right]^{2}\right\}
$$

with the summation being over the available bands, denoted here by the index $i$. The function $P(z)$ is the redshift prior, $m_{\bmod , i}$ are the model magnitudes computed from the templates (see $\S 2.2), m_{i}$ are the measured magnitudes, and $d m_{i}$ are the photometric errors and model dispersions summed in quadrature. In order to account for the different dispersions in the absolute magnitudes of the model SNe and their colors, we technically do not fit $n$ magnitudes in $n$ bands but rather one magnitude and $(n-1)$ colors. We marginalize the likelihood function over the three parameters, $z, A_{V}$, and $t$, and obtain the summed likelihood for each type of SN, commonly referred to in Bayesian literature (e.g., Gelman et al. 1995) as the "evidence,"

$$
E_{\text {type }}=\int L_{\text {type }}\left(z, A_{V}, t\right) d z d A_{V} d t
$$

In order to classify the $\mathrm{SNe}$, we define a relative evidence to express the probability that an object is a $\mathrm{SN}$ Ia as

$$
P_{\mathrm{Ia}}=\frac{E_{\mathrm{Ia}}}{E_{\mathrm{Ia}}+E_{\mathrm{CC}}} .
$$

We also derive for each $\mathrm{SN}$ its posterior redshift, assuming it belongs to a given type of $\mathrm{SN}$, by marginalizing the relevant likelihood function over the nuisance parameters, namely, the age and the extinction. The final output is then the probability for the $\mathrm{SN}$ to be of Type Ia, rather than being a core-collapse event, and its posterior $z$-pdf. In the following sections we consider the adopted type of a SN to be the type which has a probability higher than 0.5 . Naturally, the higher this probability, the more secure is the classification. We also examine the $\chi^{2}$ value for the best-fitting template in order to ascertain the goodness of fit in an absolute sense, and in order to reject SN impostors, mainly active galactic nuclei (AGNs).

\section{TESTS ON REAL DATA}

We now apply our algorithm to two real data sets, SNLS and GOODS, that have photometric data of the type we are considering (e.g., SNe observed in at least three bands) and follow-up spectroscopy based on which we can evaluate the performance of our method. 


\subsection{SNLS}

\subsubsection{Type Ia SNe}

We begin with 71 published light curves of SNe Ia from the SNLS project, ${ }^{4}$ described in Astier et al. (2006). The SNLS is a 5 yr "rolling" SN Ia survey, using the MegaCam wide-field imager on the $3.6 \mathrm{~m}$ Canada-France-Hawaii Telescope. The fields are imaged in four bands, similar to the Sloan Digital Sky Survey $g, r, i$, and $z$ (Fukugita et al. 1996; Astier et al. 2006), with limiting magnitudes in $i$ of the order of $24.5 \mathrm{mag}$. The SN candidates are confirmed using spectroscopy from 8-10 m class telescopes. The $\mathrm{SNe}$ in our sample are all spectroscopically confirmed SNe Ia at $z=0.25-1$, with a median of $z=0.6$. Typical photometric errors are smaller than $0.1 \mathrm{mag}$. Since our main focus is on classification by means of three bands, we ignore at first $g$-band data (but see $\S 4.4$ ), and use for each SN only those data combinations which have same-day $( \pm 1)$ photometry in $r$, $i$, and $z$. This produces a sample of 172 "objects," extracted from $58 \mathrm{SNe}$. This selection does not affect the redshift distribution, which remains similar to the distribution of the original sample.

Since these SNe have spectroscopic redshifts, we create simulated photometric redshifts, $z_{\text {rand }}$, by adding to each object's redshift a normally distributed (with zero mean and $\sigma_{1}$ standard deviation) error,

$$
z_{\text {rand }}=z_{\text {spec }}+\operatorname{noise}\left(\sigma_{1}\right)
$$

and create a Gaussian $z$-pdf of width $\sigma_{2}$, centered on the noiseadded redshift,

$$
P_{\text {phot }}(z)=\frac{1}{\sigma_{2} \sqrt{2 \pi}} \exp \left[-\frac{\left(z-z_{\text {rand }}\right)^{2}}{2 \sigma_{2}^{2}}\right] .
$$

To evaluate the impact of the precision of the redshift determination, we have run the $\mathrm{SN}-\mathrm{ABC}$ for various combinations of $\sigma_{1}$ and $\sigma_{2}$ values. For every $\mathrm{SN}$ we perform several specific $z_{\text {rand }}$ realizations and average the outcomes.

In Figure 1 we present the $\mathrm{SN}-\mathrm{ABC}$ output $P_{\text {Ia }}$ distributions for various precisions of redshift determinations (left panel) and the posterior redshift compared to the spectroscopic one (right panel). We start with $\sigma_{1}=0$ and $\sigma_{2}=0.01$, which can represent well-determined, i.e., spectroscopic, redshifts. In this case $\sim 97 \%$ of the SNe are correctly classified by the SN-ABC. With $\sigma_{1}=\sigma_{2}=0.1$, a reasonable precision for photometric redshifts in most surveys and redshift ranges (e.g., Grazian et al. 2006; Coe et al. 2006), this result remains unchanged, with $97 \%$ correctly classified SNe and $\sim 65 \%$ having $P_{\text {Ia }}>0.9$. The posterior redshift scatter is marginally reduced compared to the constructed prior standard deviation of $\sigma=0.1$, indicating that this precision in redshift is about the limit of what can be achieved with single-epoch data in three bands.

For $\sigma_{1}=\sigma_{2}=0.3$, i.e., very broadly distributed and imprecise redshifts and probably the worst-case scenario for photometric redshifts, we correctly classify $\sim 76 \%$ of the SNe as Type Ia and improve the scatter in the redshift determination from $\sigma=0.3$ to an a posteriori value of $\sigma \sim 0.17$, thus improving the redshift dispersion by almost a factor of 2 . On the other hand, it can clearly be seen that the posterior redshift distribution is heavily biased toward lower $z$. This is a consequence of the fact that the lower the redshift, the more freedom the minimization has in the other parameters. At lower $z$ the SN template is inherently brighter and

\footnotetext{
${ }^{4}$ Available at http://snls.in2p3.fr/conf/release/
}

can be "aged" and extinguished in order to fit the observed magnitudes, while at higher $z$ it must be closer to peak and less extinguished.

The most extreme scenario is the absence of any prior on the redshift. This is probably relevant only to the few objects that have no measured host due to its faintness. In this case we drop to only $\sim 60 \%$ correct classifications, only slightly better than random assignment of SN type. Not surprisingly, the parameter space is wide enough to accommodate both types when no information on the host redshift exists and the SN light/color curve is so scantily sampled.

We can thus conclude that single-epoch photometry in three bands, combined with a reasonably well determined host galaxy redshift, is sufficient to recognize $\mathrm{SNe}$ Ia, with only a few false negatives and high confidence levels.

\subsubsection{Type II-P SNe}

In order to measure the performance of the $\mathrm{SN}-\mathrm{ABC}$ in correctly classifying $\mathrm{CC}-\mathrm{SNe}$, we repeat the procedure described in $\S 3.1 .1$, applying the same "pseudo-photo- $z$ " method to the much smaller sample offive Type II-P SNe presented in Nugent et al. (2006). We extract 25 "objects" with same-day photometry from among three of these $\mathrm{SNe}$, which are at redshifts of $0.13-0.21$.

As shown in Figure 2, when using the precise spectral redshifts we achieve a perfect success rate with not a single object misclassified. When using broader, more realistic $z$-pdfs, we reach success rates between $85 \%\left(\sigma_{1}=\sigma_{2}=0.03\right)$ and $75 \%$ $\left(\sigma_{1}=\sigma_{2}=0.1\right)$. Since the redshifts of these $\mathrm{SNe}$ are significantly lower than those of the SNLS Ia sample, we examine cases with comparatively smaller values of $\sigma_{1}$ and $\sigma_{2}$ that are more reasonable for photometric redshifts in this range.

In all the simulated photo- $z$ schemes we have examined, for both the Ia and CC-SNe the minimum $\chi^{2}$ values are lower than the value that defines the $99 \%$ confidence interval on the goodness of fit, which we derive from simulations, as will be described in $\S 4$ below. The best-fit templates therefore fit the data well in an absolute sense.

While the tests on the SNLS samples, both Types Ia and II-P, indicate that our algorithm works well, these results may be misleading since these samples were selected by the SNLS team for their quality, and are not in any sense a complete representation of all the SNe found during the survey. The SNLS pipeline includes a color-based preselection of objects for spectroscopy, and the sample might be biased against SNe Ia with uncharacteristic colors. In order to check our method on a complete sample, we apply the SN-ABC to all of the SNe found during the Hubble Space Telescope (HST) GOODS campaign.

\subsection{GOODS}

We proceed with a SN sample from the GOODS SN survey. As part of this project, two fields of about $150 \mathrm{arcmin}^{2}$ each were observed with the ACS on HST every 45 days, over five epochs, in order to search for $\mathrm{SNe}$. We have compiled from Riess et al. (2004a) and Strolger et al. (2004) all available photometry for the $42 \mathrm{SNe}$ found in the GOODS fields. These SNe cover a redshift range of $0.2-1.55$, with a median of 0.76 , which is higher than the SNLS range. The light-curve coverage is more limited than for the SNLS sample, and not all SNe have spectroscopic redshifts or types. However, GOODS is a complete sample, without any subsequent selection. The $\mathrm{SNe}$ were classified following a decision scheme presented in Strolger et al. (2004), and the whole sample was divided into three subsets- "gold," 

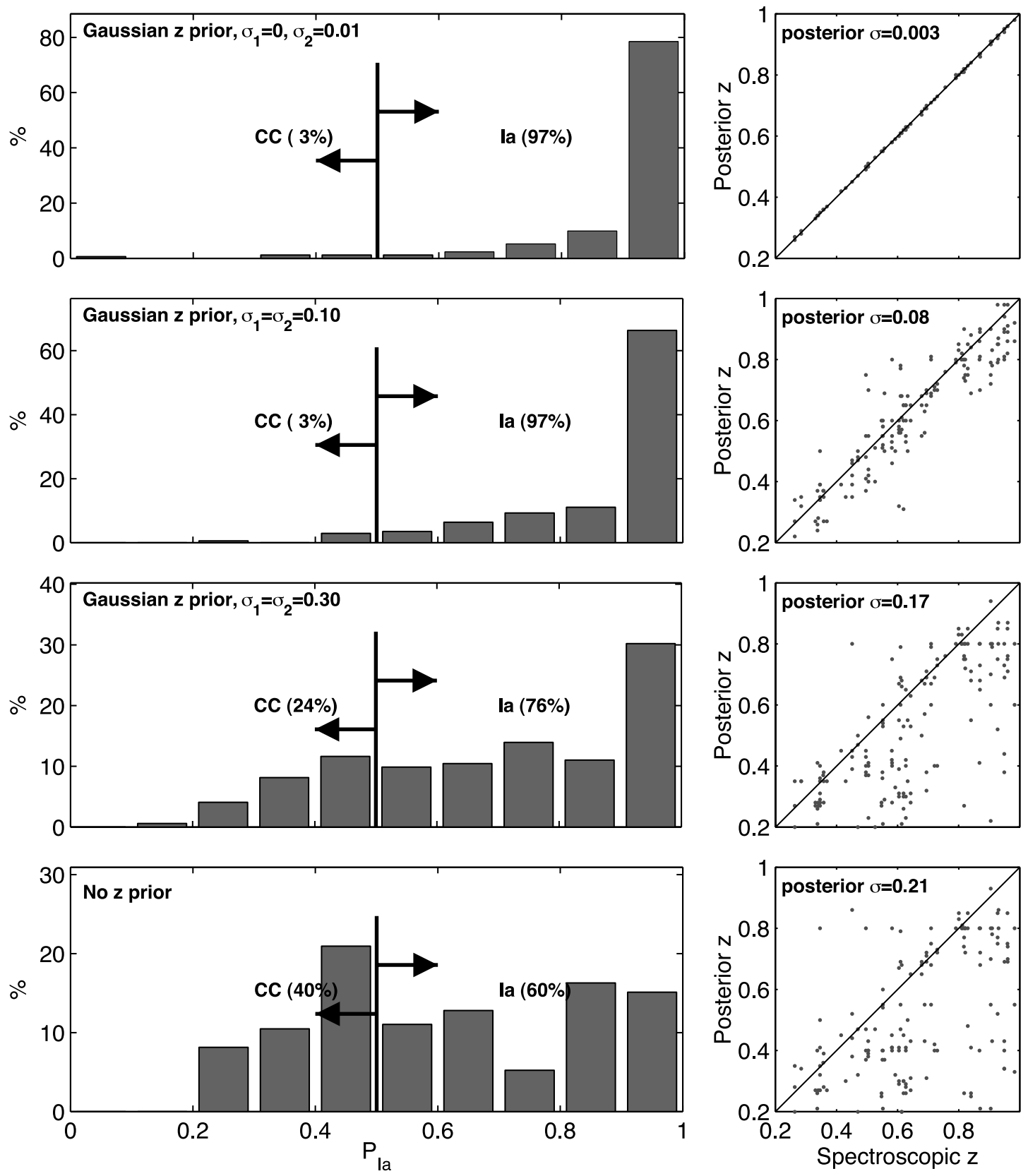

FIG. 1.-Classification of SNe Ia from SNLS. Left: Distribution of the type determination parameter $P_{\text {Ia }}$ for different redshift priors. Values of $P_{\text {Ia }}$ higher than 0.5 mean the object is probably a SN Ia, while values smaller than 0.5 mean it is a CC-SN. Right: Posterior redshifts for the priors on the left. The solid line is $z_{\text {posterior }}=z_{\text {spec }}$, for reference. The SN-ABC works well, assuming high-quality photo-z values are available (top two panels). [See the electronic edition of the Journal for a color version of this figure.]

"silver," and "bronze" - according to the reliability of the type determinations.

As in $\S 3.1$, we first reject all the $\mathrm{SNe}$ which do not have same-day $( \pm 1)$ photometry in three bands (in this case F606W, F775W, and F850LP), leaving $28 \mathrm{SNe}$. After excluding one $\mathrm{SN}$ with no redshift information, we extract from the remaining $27 \mathrm{SNe}$ (13 Ia, $14 \mathrm{CC}) 41$ same-epoch photometry "objects," of which 23 are Type Ia and 18 are CC. Among these 41 objects, 28 (17 Ia, 11 CC) have spectroscopic redshifts, while 13 (6 Ia, 7 CC) have only photometric redshifts. The requirement of same-epoch photometry changes the redshift distribution of the sample, mainly by rejecting the few $\mathrm{SNe}$ with $z>1$, and leaves a distribution with a median redshift of $\sim 0.6$, ranging from 0.2 to 1 , similar to the SNLS Ia sample.
For those SNe with spectroscopic redshifts we create pseudophoto- $z$ values, repeating the approach in $\S 3.1$, with $\sigma_{1}=\sigma_{2}=$ 0.1 . When applied to this sample, the SN-ABC correctly classifies 16 of the $17 \mathrm{SNe}$ Ia and 9 of the $11 \mathrm{CC}-\mathrm{SNe}$. The single SN Ia which is misclassified is the last of three observations of SN 2002hr, a SN Ia, about 2 months past maximum brightness. The previous two epochs of the same $\mathrm{SN}$ are well classified. The misclassified CC-SNe are SNe 2002kb and 2003bb. For $\sigma_{1}=0$, $\sigma_{2}=0.01$, representing well-determined spectral redshifts, the success rates remain unchanged for the CC-SNe, but for the $\mathrm{SNe}$ Ia one more object is misclassified, one of the three epochs of SN 2002ga, a bronze SN Ia. Since it is well classified with a broader $z$-pdf, its misclassification may be due to an underestimated redshift uncertainty. 

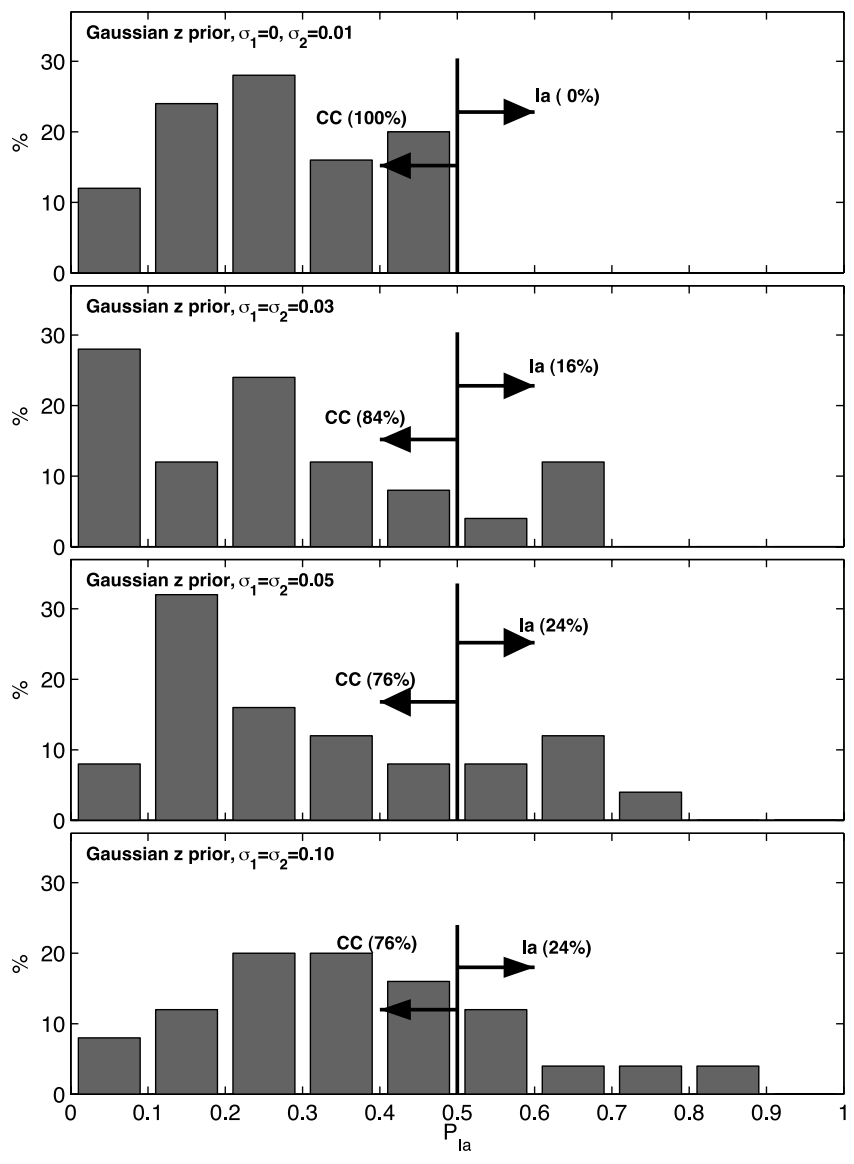

FIG. 2.- Same as Fig. 1, but for SNLS SNe II-P with four different redshift priors. [See the electronic edition of the Journal for a color version of this figure.]

For those SNe with photometric redshifts, we assume a Gaussian pdf with $\sigma_{2}=0.1$, since we do not have the real $z$-pdfs. For this photo-z sample, we recover the same classification as that of the GOODS team for only two of the six SNe Ia and four of the seven $\mathrm{CC}-\mathrm{SNe}$. Increasing $\sigma_{2}$ to a value of 0.2 to test whether we have underestimated the uncertainty in the redshift does not improve this result.

It is clear that the SN-ABC functions extremely well on the GOODS SNe with spectroscopic redshifts, mostly in accord with the classification presented in Strolger et al. (2004), while for the SNe with photo- $z$ values there are severe discrepancies. This illustrates the difficulty of classifying SNe without spectroscopy, and it is hard to ascertain which classification scheme is correct for every object. If we consider agreement between the GOODS classification and ours as an indicator of the quality of the classification itself (either the SN-ABC or GOODS), it is clear that our division of the sample according to the existence or nonexistence of spectral redshift splits the sample into distinct quality classes. This is less true for the GOODS gold/silver/bronze quality flags (although one should note that these divisions are not independent). For example, our classification of the CC-SNe with spectral redshifts, belonging to the bronze sample, is in agreement with the GOODS type for all the objects, while it is in disagreement for one of the four bronze CC-SNe with photo-z. More dramatically, for the silver $\mathrm{CC}-\mathrm{SNe}$, neither of the two SNe with photo- $z$ are similarly classified, while four out of the five with spectral redshifts are in agreement. Similar trends are seen with the Type Ia SNe. Since all the SNe Ia in the gold sample have spectral redshifts, this issue has no implications for this particular higher quality subset. We note that, by stating that the subsample with photometric redshifts is of lower quality, we do not imply that the problems arise necessarily from the photometric redshifts themselves, since we do not have the information needed to reveal the source of the discrepancies. The fact that the SN-ABC and GOODS classification for the SNe with spectra are in excellent agreement shows that our algorithm works well. The fact that there are significant discrepancies when classifying $\mathrm{SNe}$ with no spectra indicates that the GOODS classification of at least some of these objects might be erroneous. This may, alternatively, indicate a weakness in the method. If the photo- $z$ value confidence limits do not represent faithfully the true uncertainty in redshift, then our algorithm could give incorrect results. As in $\S 3.1$, there are no objects with minimum $\chi^{2}$ values outside the $99 \%$ confidence interval.

An advantage of our SN-ABC procedure is that it provides a quantitative indication of the quality of the classification, in the form of the $P_{\text {Ia }}$ value, that can be used for subsequent error estimation. The issues of classification and redshift uncertainties were addressed in Riess et al. (2004a) by deriving separate Hubble diagrams from the gold and silver samples (and ignoring the bronze sample). Dahlen et al. (2004) deal with the uncertainties in the classification only (i.e., ignoring the possible errors in redshift determination) by testing how their results on SN rates change if the entire bronze sample is misclassified. Strolger et al. (2004) used the entire GOODS sample, irrespective of quality, in their derivation of the SN Ia time delay distribution. The last treatment does not test for possible biases that arise from errors in classification or redshift determination.

\section{TESTS ON A SIMULATED SN SAMPLE}

While the GOODS sample is complete, it is quite small, and even smaller when restricted to SNe with high-confidence classification. This motivates us to explore biases that might arise from our classification algorithm, and the dependence of the success rate on various parameters, by using a large simulated sample of SNe. The simulated sample also permits us to test the performance of the algorithm when the input data are additional CC types, as well as SN “impostors" such as AGNs. In $\S 4.4$ we examine the influence on the classification of having more, or less, than three photometric bands.

We have randomly generated $5000 \mathrm{SNe}$ of each of the SN types: Ia, Ibc, II-P, and IIn. To each SN we assign a redshift, an epoch, and a host extinction. These characteristics are drawn from a uniform distribution. While these are not the true distributions, intrinsic or observed, they hold two advantages for our objectives. First, flat distributions facilitate the discovery of possible trends in the reliability of the SN-ABC classification by creating many SNe with every combination of properties. Second, the true distributions of the SN rates as a function of redshift, type, extinction, etc., are yet unknown, or highly uncertain. Assuming particular distributions could introduce unknown systematics into the comparative success rates. The results could also be used incorrectly to draw, for example, the overall contamination of a SN Ia sample by CC-SNe. The contamination fraction, while very valuable for many SN searches, depends strongly on the intrinsic rates of the different types of CC-SNe as a function of redshift. Such an estimate of contamination must therefore be tailored to a specific survey, taking into consideration the range of possible distributions in $\mathrm{SN}$ properties.

For comparison of the results based on our simulated $\mathrm{SNe}$ to those based on the real SN samples, we mimic the observational properties of the GOODS sample with its three HST bandpasses, photometric errors and limiting magnitudes. The results in this 

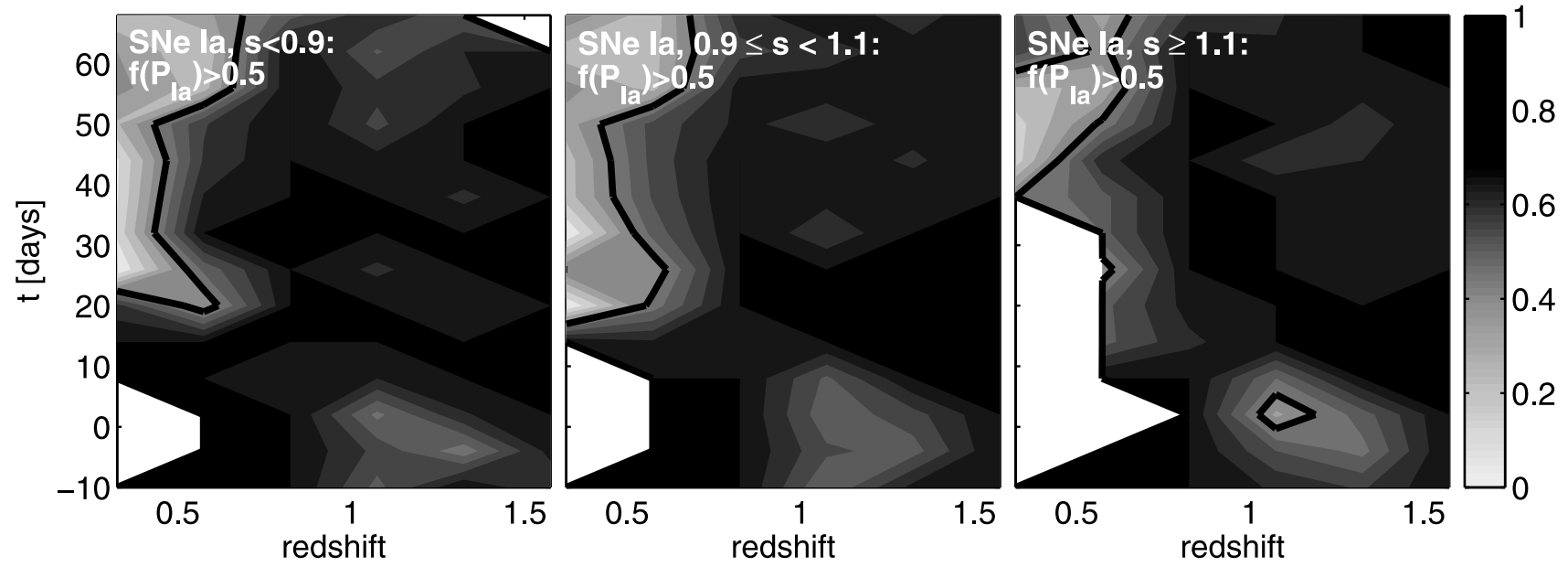

FIG. 3.-Classification success rate for simulated Type Ia SNe with three stretch ranges, as marked, as a function of redshift and age. Contours trace $f\left(P_{\text {Ia }}>0.5\right)$, the

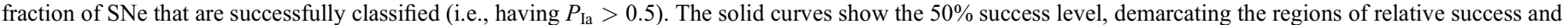

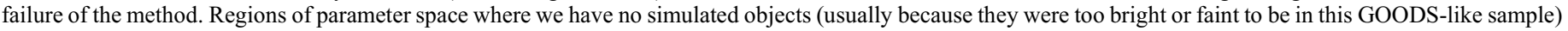

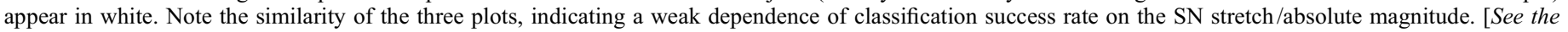
electronic edition of the Journal for a color version of this figure.]

section are for this specific configuration and serve as an illustration of the capabilities of the SN-ABC.

As in $\S 2.2$, we calculate the synthetic magnitudes of the fake SNe using the SEDs from the spectral templates of Nugent et al. (2002) for Types Ia, Ibc, and II-P SNe. Since the Nugent et al. (2002) spectra of Type IIn SNe are theoretical blackbody SEDs, for this type only we use the templates from Poznanski et al. (2002). As in $\S 2.2$, absolute magnitudes and their dispersions are taken from Dahlen et al. (2004). The scatter in color within each type is applied to the CC-SNe by adding an intrinsic, normally distributed noise with a standard deviation of $\sigma=0.2 \mathrm{mag}$, the value used by Sullivan et al. (2006a). The SNe Ia we simulate are given different stretches $s$, following the method described in Sullivan et al. (2006a). We simulate a Gaussian distribution of stretches with an average of $s=1$ and a dispersion of $\sigma=0.25$, truncated in the range $0.6 \leq s \leq 1.4$. We model the stretchluminosity relation using the formalism $M_{B c}=M_{B}-\alpha(s-1)$ (Perlmutter et al. 1999), where $M_{B C}$ and $M_{B}$ are the corrected and uncorrected $B$-band absolute brightnesses, respectively, and the correlation factor is $\alpha=1.47$. We also apply color-stretch corrections using the method presented in Knop et al. (2003) by dividing the template spectra of normal, $s=1 \mathrm{SNe}$ Ia by smooth spline functions, in order to match their rest-frame $U B V R I$ colors to those of $\mathrm{SNe}$ with various stretches. We have measured the mean photometric errors of the GOODS sample in each band as a function of magnitude and, again assuming the noise is normally distributed, have added it to each object. We further assigned to the SNe a pseudo-photo- $z$ with $\sigma_{1}=\sigma_{2}=0.1(\operatorname{see} \S 3.1 .1)$.

\subsection{Type Ia SNe}

Figure 3 shows the average success rate of our algorithm, as a function of SN age and redshift, for three different stretch ranges. Each panel shows in contours the fraction of SNe Ia that are correctly classified by the $\mathrm{SN}-\mathrm{ABC}$, i.e., the fraction of $\mathrm{SNe}$ that are given $P_{\text {Ia }}$ values higher than half. The solid curves show the $50 \%$ success level, demarcating the regions of relative success and failure of the method. The regions of parameter space where we have no simulated objects (usually because they were too bright or faint to be in this GOODS-like sample) appear in white. As can be seen, the differences in plots for different stretch ranges are minor, showing that the diversity in color and magnitude among SNe Ia does not affect the classification significantly. In the subsequent analysis, we marginalize over the full range of stretch values.

As can be seen in the top left panel of Figure 4 (which is basically a weighted sum of the three panels of Fig. 3), the $\mathrm{SN}-\mathrm{ABC}$ success rate on simulated $\mathrm{SN}$ Ia data is high, greater than $90 \%$ in most regions of parameter space. The notable exception to this success is the population of 1-2 month old, relatively low $-z$ SNe. As already shown in Poznanski et al. (2002), such SNe Ia have blue rest-frame colors that are similar to those of CC-SNe. Furthermore, the older the SN, the fainter it is, and therefore it can be erroneously fitted more easily with younger CC-SN templates, which are intrinsically dimmer. This means that by excluding objects from a sample below a certain $z$, one can obtain higher classification success rates. Figure 5 (top left) shows, for the same simulated $\mathrm{SNe}$ Ia, the average $P_{\text {Ia }}$ value (rather than the success rate) as a function of age and redshift. Clearly, not only are most of the SNe classified correctly, but their classification has a high confidence, with an average $P_{\text {Ia }}$ greater than 0.8 in most regions. We also note, from a comparison of the top left panels of Figures 4 and 5, that the average $P_{\text {Ia }}$ value follows quite well the actual success rate, meaning that it can serve as a reliable quality indicator. We have searched for trends in the success rates of the classification as a function of all the other parameters, such as color, photometric errors, and extinction, and found no obvious correlations.

\subsection{Core-Collapse SNe}

In the three remaining panels of Figure 4 we can see that the picture is more complex within the zoo of CC-SNe. Most Type II-P SNe are correctly classified, being progressively better classified at later times. This is the complementary image of what we see with Type Ia SNe. The colors of young II-P SNe resemble those of Ia SNe about a month past maximum light. For this reason Type II-P SNe are also better classified at low $z$.

The SN-ABC fails with Type IIn SNe, with about half of them being erroneously classified as SNe Ia. This is the result of a degeneracy in color-magnitude space between these two types, and for which we have no straightforward solution. As already noted in $\S 2.2$, we do not use in the SN-ABC a set of Type IIn templates, because their addition, while improving the classification 

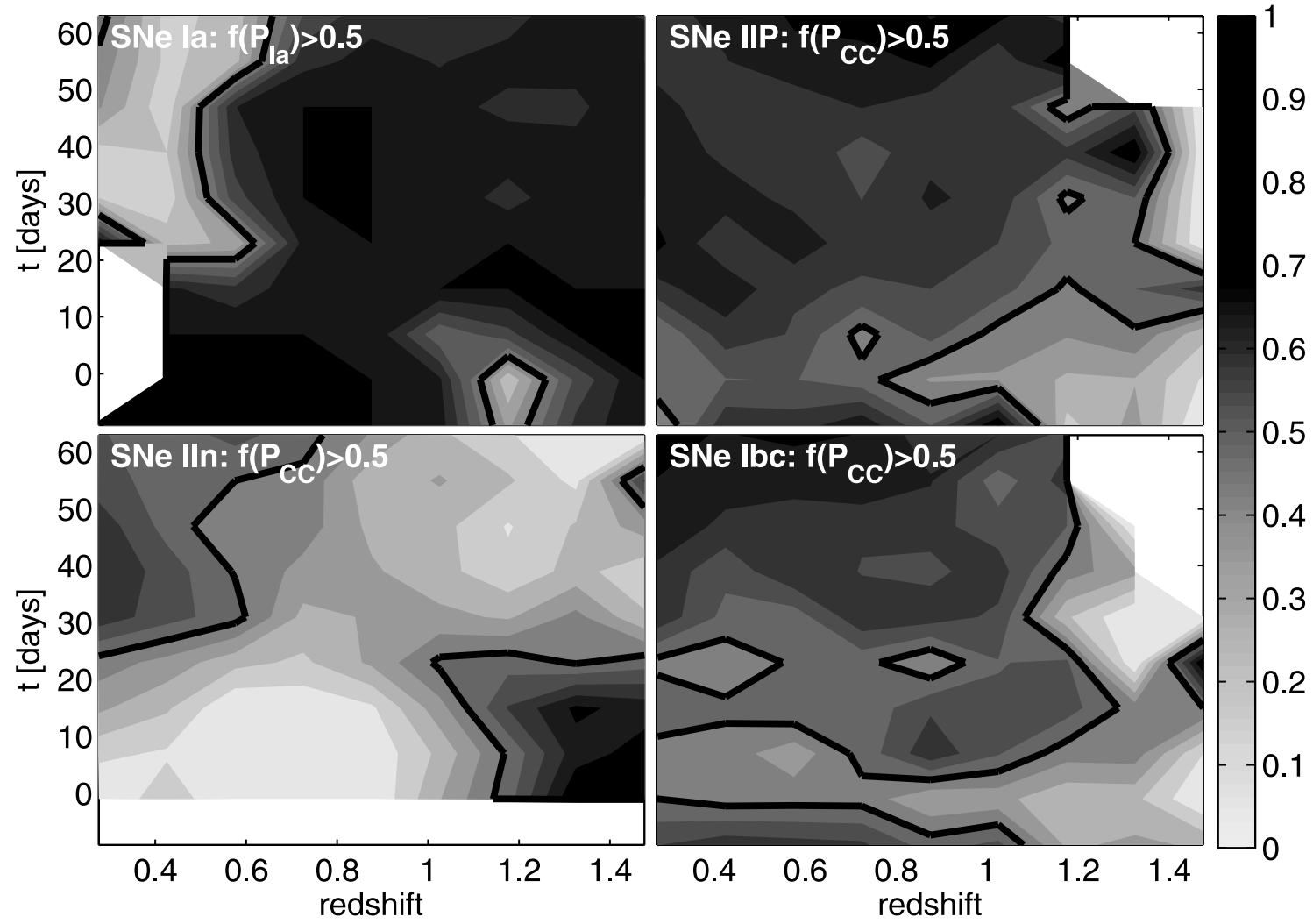

Fig. 4.-Same as Fig. 3, but for simulated SNe of Types Ia, II-P, Ibc, and IIn, as marked. The top left panel is effectively the weighted sum of the three panels of Fig. 3. Types II-P and Ibc are correctly classified as CC-SNe for a large range in age and redshift, but SNe IIn are generally misclassified as SNe Ia. [See the electronic edition of the Journal for a color version of this figure.]
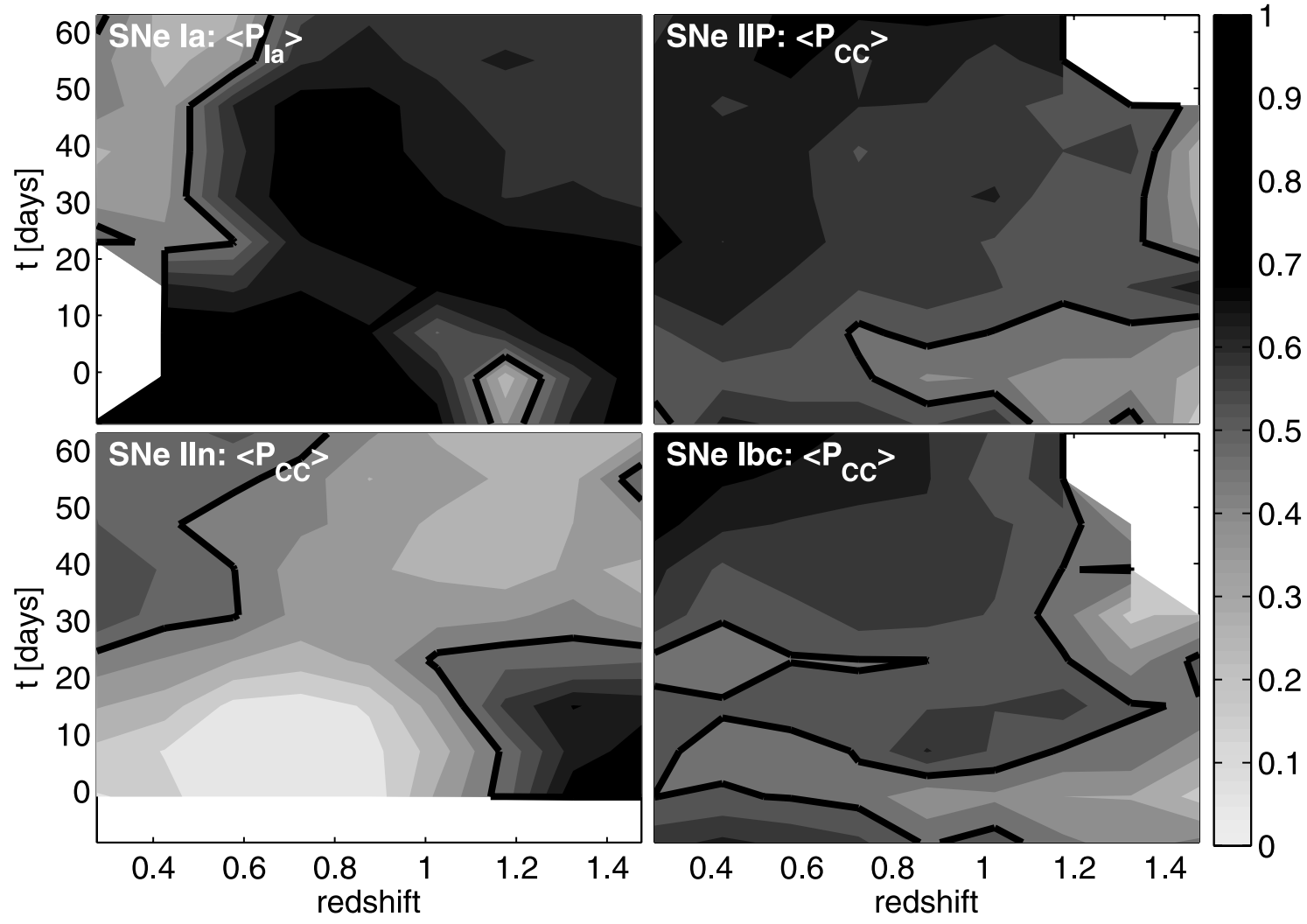

FIG. 5.-Same as Fig. 4, but plotting contours of mean $P_{\mathrm{Ia}}$ (top left) or $P_{\mathrm{CC}}$ (top right, bottom) values for simulated SNe of Types Ia, II-P, Ibc, and IIn, as marked. The solid curves show the $P=0.5$ level. Note the similarity with Fig. 4 , indicating that the SN-ABC output, $P_{\mathrm{Ia} / \mathrm{CC}}$, is a good indicator of the reliability of the classification. [See the electronic edition of the Journal for a color version of this figure.] 
of SNe IIn as CC-SNe, would considerably hinder SN Ia classification. Type IIn SNe likely result from the core collapse of very massive stars (Gal-Yam et al. 2007), the high-mass tail of the initial mass function, and are therefore suppressed in volumelimited samples. Cappellaro et al. (1997) measure their fraction to be $\sim 2 \%-5 \%$ of the $\mathrm{CC}$ population in the local universe. Nonetheless, due to their brightness, in magnitude-limited samples their fraction could be as high as $\sim 15 \%-20 \%$. For example, in IAU circulars between 2005 January and 2006 September, the Nearby Supernova Factory (Wood-Vasey et al. 2004) and the SDSS II SN survey (Dilday et al. 2005) have together reported $\sim 11$ Type IIn SNe out of $\sim 55 \mathrm{CC}$-SNe. SNe IIn appear to be an important contaminant of future SN Ia samples, and may cause difficulties for cosmology-oriented studies. This may have already affected some current studies that relied to some degree on photometric classification, e.g., Barris \& Tonry (2006). Such contamination could explain their measured SN rates, which seem inconsistent with other published results, as discussed by Neill et al. (2006). The possible contamination of SN Ia samples by Type IIn SNe has also been discussed by Germany et al. (2004).

Our algorithm correctly classifies most Type Ibc $\mathrm{SNe}$ as $\mathrm{CC}-\mathrm{SNe}$, but with lower success rates than Type II-P SNe. Considering the fact that we are using Type II-P templates to recognize Type Ibc events, this should not be surprising. As with $\mathrm{SNe}$ II-P, when $\mathrm{SNe}$ Ibc are near peak, their colors are degenerate with those of SNe Ia. A caveat however, is that the templates from which we have simulated the Type Ibc SNe may not truly represent the high- $z$ population, a possibility that must await future spectroscopy of such events. As was the case for SNe Ia, we find no trends in the SN-ABC results as a function of all the other simulated parameters, such as magnitudes in the different bands, photometric errors, and extinction. The top right and two bottom panels of Figure 5 show that for CC-SNe, as was the case for SNe Ia, the average $P_{\mathrm{CC}}$ values follow the success rate of the SN-ABC; when the success rate is low, the $P_{\mathrm{CC}}$ values are, on the average, also lower, indicating when the fit is poor and thus serving as measures of quality for the classification of each object.

In order to simulate rolling surveys, where a field is imaged repeatedly and the $\mathrm{SNe}$ are found when they are young, we have run simulations using only $\mathrm{SNe}$ younger than 3 weeks past explosion. Assuming a young SN age during classification, i.e., marginalizing the likelihood only over the relevant extent in age, improves the success fractions of the SN-ABC significantly, as expected, since it removes many of the young versus old degeneracies. The improvement depends on the redshift range and SN type. For example, for redshifts between 0.4 and 0.6 , the success fraction for SNe Ia improves from $91 \%$ to $93 \%$.

\subsection{Misclassification and Contamination by AGNS}

The fractional contamination by false positives (e.g., non-Ia types among $\mathrm{SNe}$ classified as Ia) will depend on the intrinsic distribution among types in a given survey, which depends on the depth of the survey. For example, shallow, flux-limited SN surveys at $z \sim 0.1$ find, following spectroscopic confirmation, an $80 \%$ Ia fraction (A. Gal-Yam et al. 2007, in preparation). Since this fraction is based on a small number of $\mathrm{SNe}$, we assume it could be as low as $50 \%$. We also assume for simplicity that the CC-SNe are equally divided between the three subtypes: II-P, IIn, and Ibc. The following numbers depend weakly on this assumption. Averaging over CC-SN subtypes, about $30 \%$ of the $\mathrm{CC}-\mathrm{SNe}$ in this redshift range $(z \sim 0.1)$ are misclassified as Ia, while about $90 \%$ of the Ia types are correctly classified as such.

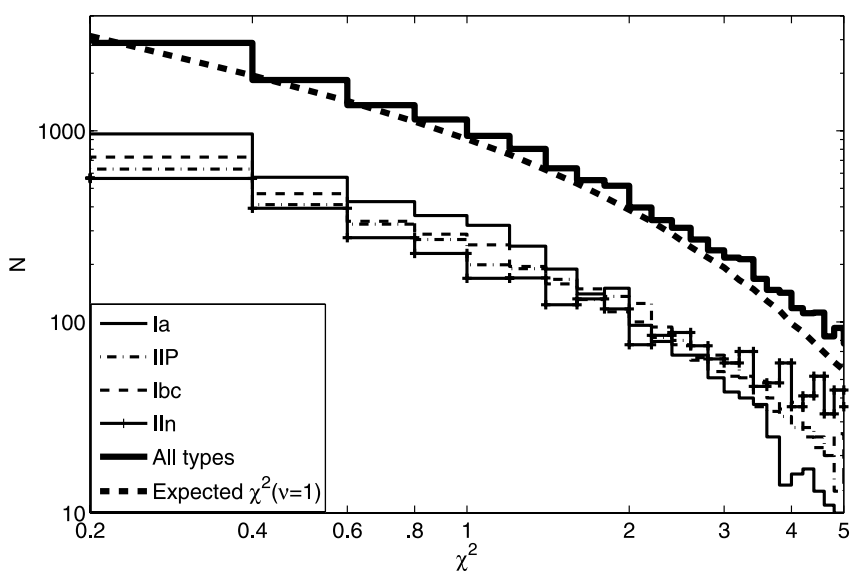

FIG. 6.-Measured $\chi^{2}$ distributions for the sample of simulated SNe, by type, and for all types combined (thick solid histogram). The thick dashed curve is the expected distribution for 1 degree of freedom, normalized to the total number of simulated objects, and matches well the measured curves.

This gives a fraction of false positives among the SNe Ia in a photometrically classified survey to this depth between $(0.3 \times$ $0.2) /(0.3 \times 0.2+0.9 \times 0.8)=0.08$ and $(0.3 \times 0.5) /(0.3 \times 0.5+$ $0.9 \times 0.5)=0.25$. Alternatively, in deep surveys such as GOODS, about $50 \%$ of the $\mathrm{SNe}$ are $\mathrm{CC}$ at $0.3 \lesssim z \lesssim 0.9$, and the misclassified CC fraction is $\sim 35 \%$. The $\mathrm{SNe}$ Ia are mostly at $0.5 \lesssim$ $z \lesssim 1.2, \sim 90 \%$ of which are correctly classified; hence, the contamination fraction is slightly higher, on the order of $(0.35 \times$ $0.5) /(0.35 \times 0.5+0.9 \times 0.5)=0.28$. In the absence of detailed knowledge about the intrinsic SN type and redshift distributions in a survey, the errors and biases introduced by misclassification are best estimated using simulations that test the ranges of intrinsic distributions that could plausibly produce the actually observed, photometrically typed distribution.

We have calculated for each of the simulated SNe the $\chi^{2}$ value for the best-fitting SN template. The $\chi^{2}$ distribution for each of the SN types can be seen in Figure 6. The distribution is similar to the expected $\chi^{2}$ probability density function with 1 degree of freedom. Had our model been linear, and its parameters nondegenerate, we would have expected to have 0 degrees of freedom, since we fit three parameters (redshift, age, and extinction) to three data points per object (the magnitudes in three bands). Fortunately, our model is nonlinear and has some degeneracies between the parameters, so that useful information can be extracted, as we have shown. From the distribution of $\chi^{2}$ values in the simulations, we can derive the $99 \%$ confidence interval, which can be used to reject objects which are poorly fit by all of our models. Such objects could be peculiar types of SNe or SN impostors (e.g., AGNs, the major contaminant in SN surveys) with significantly different colors. As already noted, in SNLS and GOODS, all the SNe we examined had $\chi^{2}$ values smaller than the $99 \%$ confidence limit of the simulated data set, meaning there were no true $\mathrm{SNe}$ that were misclassified as peculiar/ impostor SNe. In our application of SN-ABC to a real "spectroscopically blind" SN survey, we can use the $\chi^{2}$ criterion to assess the contamination by peculiar events and by AGNs.

Using the same approach used to simulate a SN sample, we have generated 400 mock AGNs using as a template the composite AGN spectrum from Vanden Berk et al. (2001) and a redshiftdependent luminosity function from Boyle et al. (2000). We have extrapolated the function to low luminosities based on the evidence presented in Wolf et al. (2003). We expect, at least in some cases, the photometric redshift to fail due to the contribution of the AGN to the colors of the host galaxy. As an approximation of 

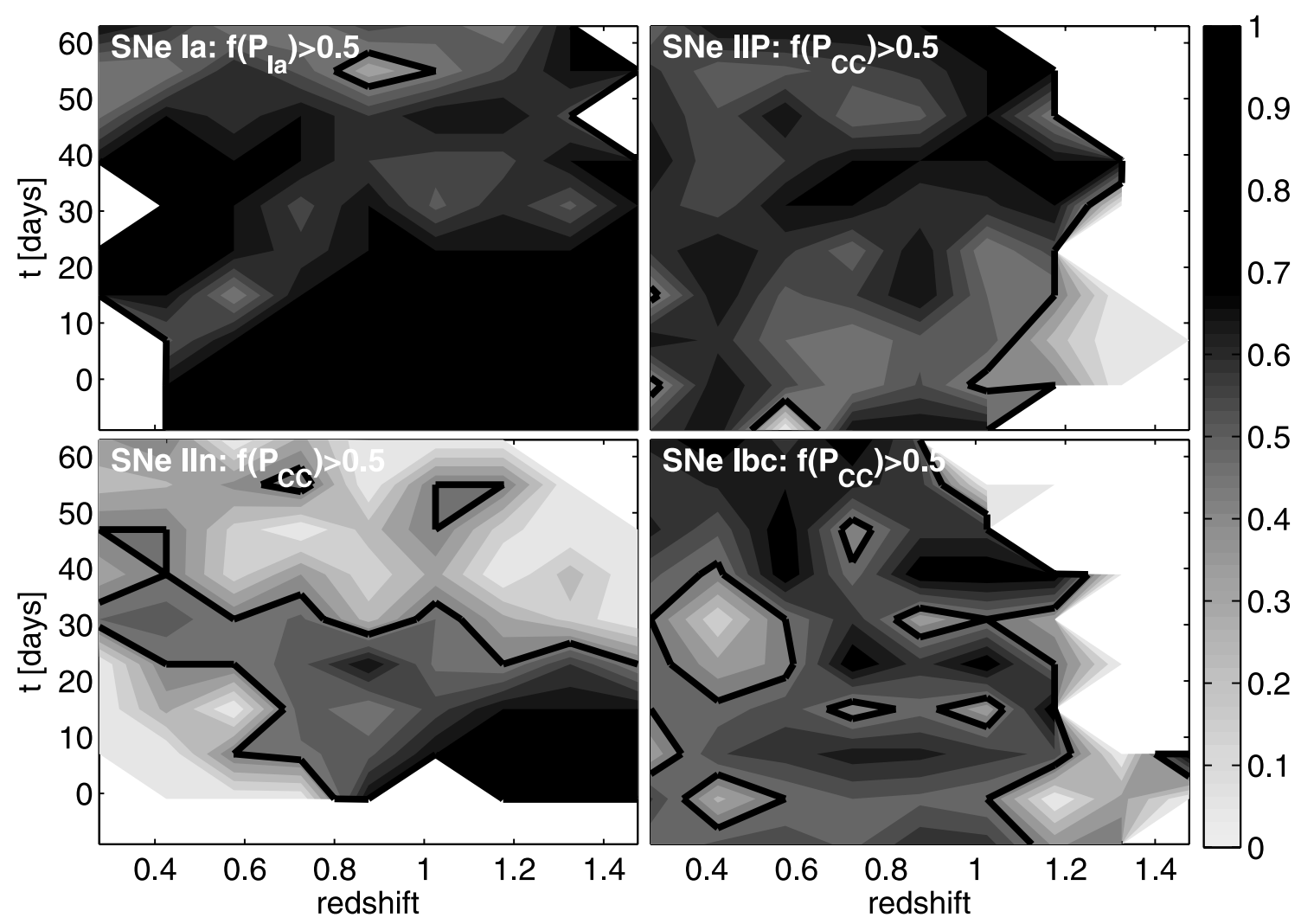

FIG. 7.- Same as Fig. 4, but for simulated SNe observed in four bands rather than three. Four bands improve the classification success rates of old, low- $z$ SNe Ia but have little effect on the success of CC-SN classification. [See the electronic edition of the Journal for a color version of this figure.]

this effect, we generate for each AGN a random host galaxy drawn from a Schechter distribution (Schechter 1976). If the AGN luminosity is smaller than the galaxy's, we assume the photo- $z$ works well, and otherwise, we generate a completely random photo- $z$. Among the AGNs, 265 are first classified as CC-SNe according to their $P_{\mathrm{Ia}}$ values, and the remaining 135 as SNe Ia. After applying a $\chi^{2}$ cut, at the $95 \%$ confidence limit, 189 of the CC impostors are rejected, but only eight of the Ia impostors. There is no significant difference between the samples with "good" and "bad" photometric redshifts, so our approximation for the redshift determination is inconsequential.

Thus, we can reject more than half of the AGNs we simulate. The remaining objects contaminate in about equal fractions the Ia and CC-SN samples. Note that in any search that includes more than two epochs, most of these AGNs will be rejected due to their non-SN-like variability, and survey-specific simulations can be carried out to test for the existence of residual contamination.

\subsection{Number of Photometric Bands}

So far, we have studied the performance of the SN-ABC when classifying $\mathrm{SNe}$ with single-epoch photometry in three bands. We now examine the effect of using more or fewer bands on the classification success rate.

We begin with the addition of observations in a fourth, bluer band, specifically the HST F475W filter. We repeat the simulations presented in the previous sections, although with fewer $\mathrm{SNe}$ (500 of each type), due to computational limitations. Comparing the results shown in Figure 7 to those obtained with three bands in Figure 4, one can see that although there is some improvement, it is not striking. The overall success rate for SNe Ia (top left panel in each) improves marginally, mainly through a better classification of 1-2 month old SNe at low redshift. How- ever, as can be seen in the remaining panels of Figure 7, for the $\mathrm{CC}$-SNe of the different subtypes, the improvement is less significant, and is null for Type IIn SNe.

In order to test whether this improvement remains marginal for real data, we used the SNLS sample, which has $g$-band observations for some of the $\mathrm{SNe}$. We repeated the process described in $\S 3.1 .1$ with the fourth band added and $\sigma_{1}=\sigma_{2}=0.1$. In this case $99 \%$ of the Ia SNe are correctly classified (only one misclassified), with $74 \%$ having $P_{\text {Ia }}>0.9$. While this is better than with three bands, it is not a dramatic improvement. For $\sigma_{1}=\sigma_{2}=0.3$, the correctly classified fraction of SNe Ia rises from $76 \%$ with three bands to $84 \%$ with four. The SNe II-P are only marginally better classified, but the sample with observations in four bands consists of only eight such objects. We thus conclude that, when the photometric redshifts are reasonably good, observations in a fourth band, for the sake of classification purposes, may be an inefficient use of telescope time.

Next, we examine the success rate of the SN-ABC when using observations only in two bands. Since the best bands for high- $z$ SN observations are the reddest, we discard the bluest band, and remain with F775W and F850LP. As expected, with two bands the results, shown in Figure 8, are degraded. Nonetheless, in some regions of parameter space the success rate of the $\mathrm{SN}-\mathrm{ABC}$ is nonnegligible. SNe Ia show the same change as when dropping from four bands to three. The success rates drop especially at low redshift and for old ages. On the other hand, surprisingly, the success rate for II-P and Ibc SNe is usually higher with two bands than with three. This could be understood in the following way. As described in $\S 2.2$, our model for the absolute magnitude of CC-SNe includes a much greater dispersion than that of SNe Ia, in order to account for their intrinsic diversity. This entails that $\mathrm{CC}-\mathrm{SNe}$ can populate wider volumes of color-magnitude 

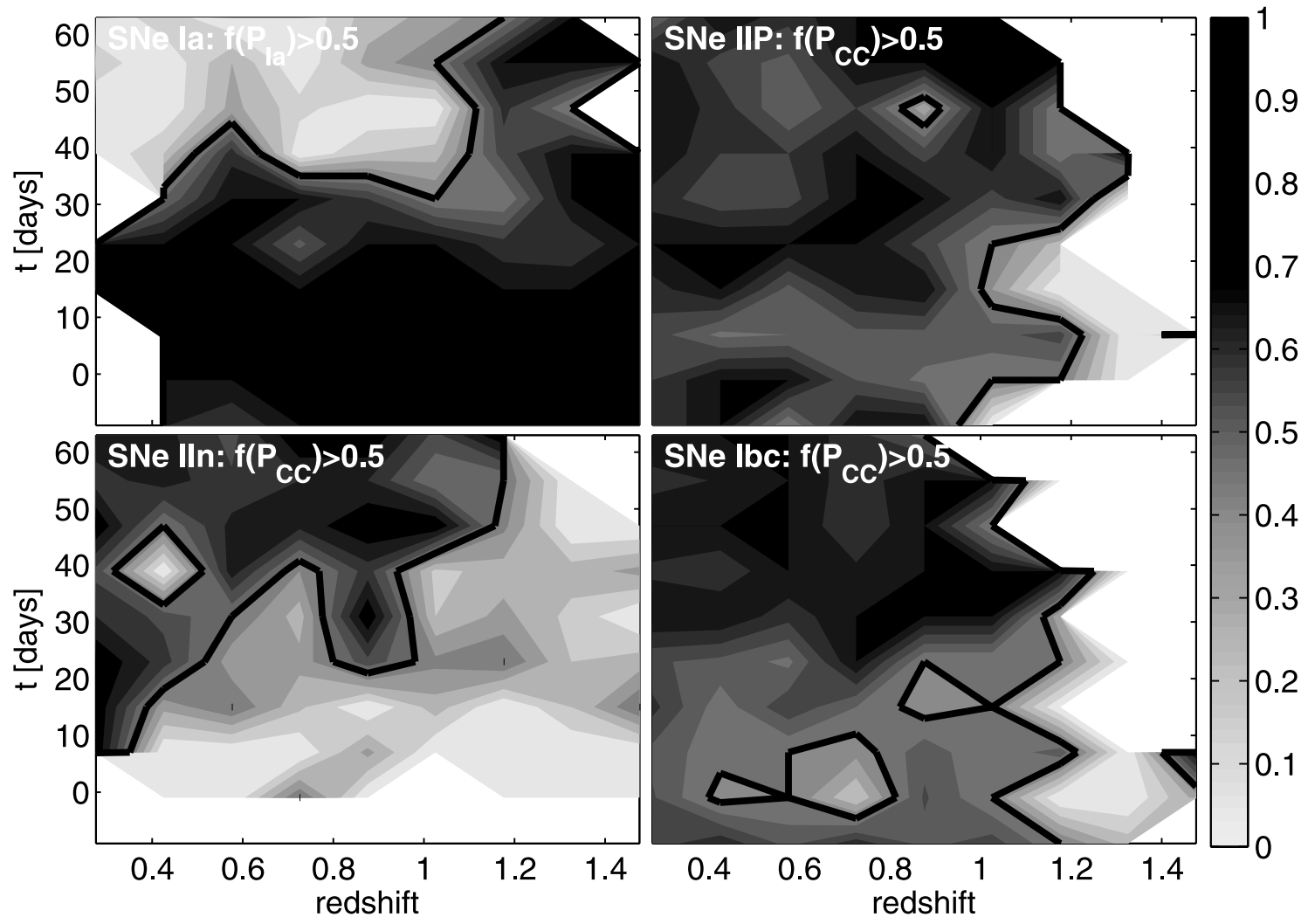

Fig. 8. - Same as Fig. 4, but for simulated SNe observed in only two bands (F775W and F850LP). As expected, SN Ia classification success is reduced significantly. For CC-SNe, classification success rates are somewhat higher than with three bands, as explained in $\S 4.4$. [See the electronic edition of the Journal for a color version of this figure.]
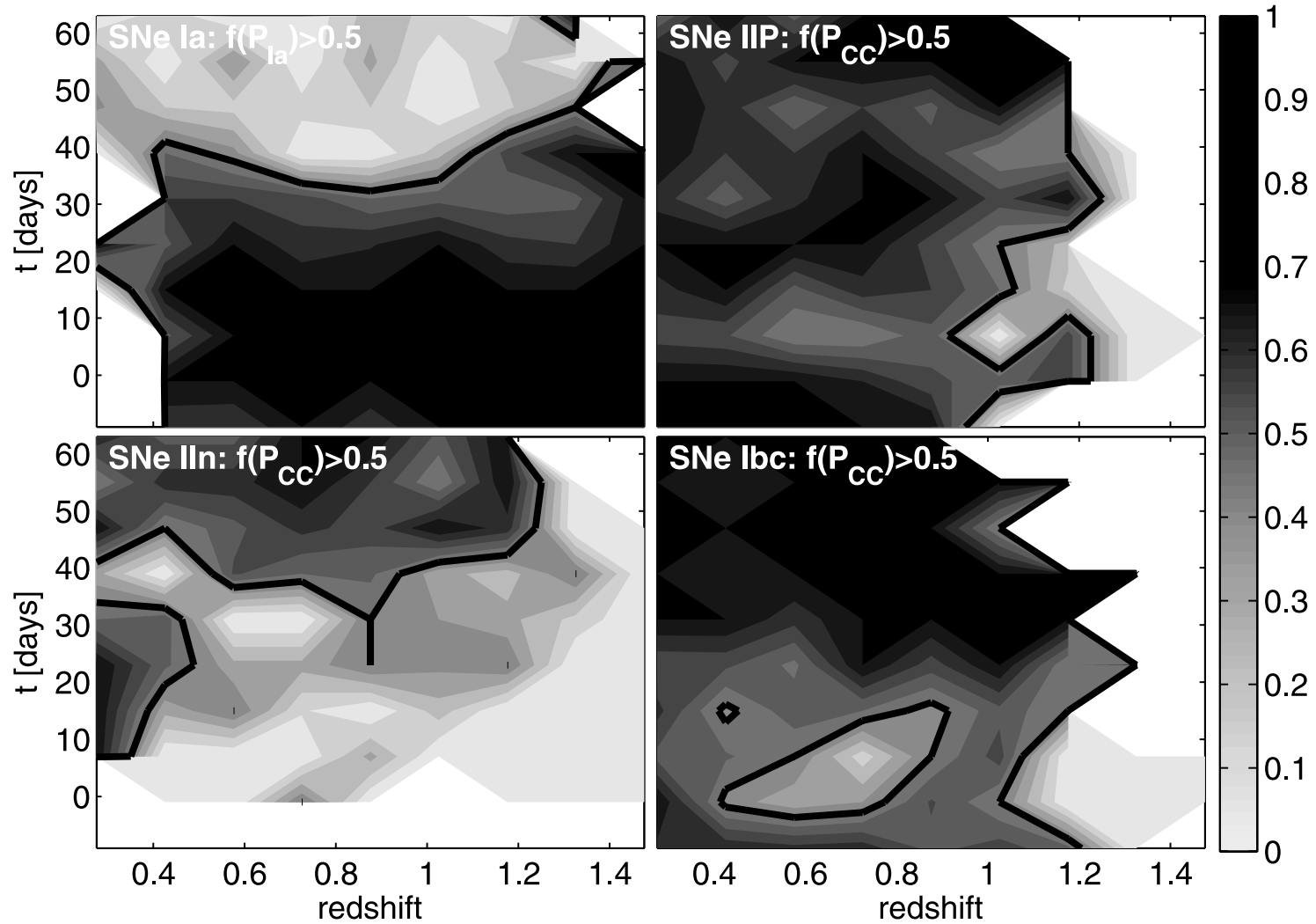

FIG. 9.-Same as Fig. 4, but for simulated SNe observed in just one band (F850LP). [See the electronic edition of the Journal for a color version of this figure.] 
space, so that when observational constraints are weak (with one or two bands observed), summing the likelihood over all parameter space makes the evidence $E_{\mathrm{CC}}$ greater on average than $E_{\mathrm{Ia}}$, and thus increases $P_{\mathrm{CC}}$ for all objects, regardless of their real type. As a consequence, $\mathrm{SN}$ Ia classification is hindered, while $\mathrm{CC}-\mathrm{SNe}$ are apparently better classified. We have tested this explanation by reducing artificially the dispersion of CC-SNe in our model. As a consequence, SN Ia classification improved dramatically, regardless of the number of observed bands, while $\mathrm{CC}-\mathrm{SN}$ classification success was correspondingly reduced, being worst with the least number of bands.

Even with only one observed band (the reddest, F850LP), as can be seen in Figure 9, the SN-ABC manages to classify successfully some $\mathrm{SNe}$, e.g. $75 \%-85 \%$ of all the SNe with redshifts $z>0.7$, excluding SNe IIn. This is mainly due to the fact that, at least in our models, SNe Ia are significantly brighter than most CC-SNe, which is enough to differentiate (probabilistically) between Ia and CC-SNe when the redshift is constrained.

Finally, AGN rejection, while improving only marginally when adding a fourth band, is weakened considerably when discarding information in one or two bands. This comes from the longer tail of high values that the $\chi^{2}$ distributions have in these cases. Such a tail increases the $95 \%$ confidence limit by a factor of 10 , when compared to the scenarios with three or four bands, and does not allow the rejection of any AGNs.

Thus, while some classification can be done with one or two photometric bands, the biases in the resulting samples are stronger and the success rates are rather low. On the other hand, four bands seem not to be worth the extra observing time over observations in three bands, if the sole purpose is to classify the SNe based on single-epoch data. This also suggests that, with better sampled light curves, the added value of a blue fourth band is small.

\section{CONCLUSIONS}

Using the SN-ABC, a Bayesian algorithm, we have studied the feasibility of a probabilistic automatic classification of $\mathrm{SNe}$, based solely on single-epoch multiband photometry and prior redshift information. While photometrically classified samples are far from competing in precision with spectroscopic samples, they could be an important source of information on the rates and statistical properties of SNe. We have tested our technique on samples of SNe from the SNLS and GOODS projects, which have spectroscopic classification, and on artificial data sets. For most samples and settings our method is promising, with high success rates. When assuming reasonably well-measured pho- tometric redshifts, the SN-ABC correctly classifies $97 \%$ of the Type Ia SNe from SNLS, and 85\% of the Type II-P SNe. Similar numbers are achieved for a subset of the GOODS SNe for which there are spectroscopic redshifts. In artificial samples, we have shown that SNe Ia are best recognized at $z \gtrsim 0.6$, or when near maximum, with success rates on the order of $95 \%$. CC-SNe are best classified several weeks after maximum, or at $z \lesssim 0.6$. Using the artificial samples, we have shown that when taking into account the whole zoo of CC-SNe, complications do arise. Specifically, Type Ibc SNe are not as well classified, and Type IIn $\mathrm{SNe}$ are usually misclassified. For surveys where $\mathrm{SNe}$ are found young, classification success fractions improve by a degree dependent on the SN type and redshift. The fractional contamination of a single-epoch photometrically selected sample of SNe Ia by CC-SNe could vary between less than $10 \%$ and as much as $30 \%$. The errors and biases introduced by misclassification are best estimated using simulations that test the ranges of intrinsic distributions that could plausibly produce the actually observed, photometrically typed, distribution.

An additional feature of the $\mathrm{SN}-\mathrm{ABC}$ is the ability to reject SN "impostors," mainly AGNs, using $\chi^{2}$ statistics. In our simulations half of the AGNs we simulated were rejected when using a $\chi^{2}$ confidence limit of $95 \%$. While we have focused on $\mathrm{SNe}$ observed in three bands, we have found that a fourth band may be of little additional benefit for the purpose of classification, while with fewer bands some classification is still possible. AGN rejection, however, fails with fewer than three bands.

In all the cases studied, the output values that we use to classify the $\mathrm{SNe}, P_{\mathrm{CC}}$ and $P_{\mathrm{Ia}}$, are also indicative of the quality of the fit and the reliability of the result. The mean values of $P_{\mathrm{CC}}$ and $P_{\mathrm{Ia}}$ track the success rates of the $\mathrm{SN}-\mathrm{ABC}$, and can be used both to define subsamples with lower uncertainties and to estimate confidence intervals. Methods such as these, and their future refinements, are a prerequisite for pushing the exploration of the $\mathrm{SN}$ population to fainter magnitudes and higher redshifts. A public release version of the SN-ABC is in preparation.

We thank the SNLS team, specifically R. Pain and P. Nugent, for access to SNLS data and valuable comments and S. Leach, E. O. Ofek, and K. Sharon for useful discussions. A. G. acknowledges support by NASA through Hubble Fellowship grant HSTHF-01158.01-A awarded by STScI, which is operated by AURA, Inc., for NASA under contract NAS5-26555.
Aldering, G. 2005, NewA Rev., 49, 346

Astier, P., et al. 2006, A\&A, 447, 31

Barris, B. J., \& Tonry, J. L. 2004, ApJ, 613, L21 2006, ApJ, 637, 427

Benítez, N. 2000, ApJ, 536, 571

Boyle, B. J., Shanks, T., Croom, S. M., Smith, R. J., Miller, L., Loaring, N., \& Heymans, C. 2000, MNRAS, 317, 1014

Cappellaro, E., Turatto, M., Tsvetkov, D. Y., Bartunov, O. S., Pollas, C., Evans, R., \& Hamuy, M. 1997, A\&A, 322, 431

Cappellaro, E., et al. 2005, A\&A, 430, 83

Cardelli, J. A., Clayton, G. C., \& Mathis, J. S. 1989, ApJ, 345, 245

Coe, D., Benítez, N., Sánchez, S. F., Jee, M., Bouwens, R., \& Ford, H. 2006, AJ, 132,926

Dahlén, T., \& Fransson, C. 1999, A\&A, 350, 349

Dahlén, T., et al. 2004, ApJ, 613, 189

Dilday, B., et al. 2005, BAAS, 37, 1459

Elmhamdi, A., Chugai, N. N., \& Danziger, I. J. 2003, A\&A, 404, 1077

Filippenko, A. V. 1997, ARA\&A, 35, 309

\section{REFERENCES}

Filippenko, A. V., Li, W. D., Treffers, R. R., \& Modjaz, M. 2001, in IAU Colloq. 183, Small Telescope Astronomy on Global Scales, ed. B. Paczynski, W.-P. Chen, \& C. Lemme (ASP Conf. Ser. 246; San Francisco: ASP), 121

Förster, F., Wolf, C., Podsiadlowski, P., \& Han, Z. 2006, MNRAS, 368, 1893

Fukugita, M., Ichikawa, T., Gunn, J. E., Doi, M., Shimasaku, K., \& Schneider, D. P. 1996, AJ, 111, 1748

Gal-Yam, A., Cenko, S. B., Fox, D. W., Leonard, D. C., Moon, D.-S., Sand, D. J., \& Soderberg, A. M. 2005, in ASP Conf. Ser. 342, 1604-2004: Supernovae as Cosmological Lighthouses, ed. M. Turatto et al. (San Francisco: ASP), 305 Gal-Yam, A., \& Maoz, D. 2004, MNRAS, 347, 942

Gal-Yam, A., Poznanski, D., Maoz, D., Filippenko, A. V., \& Foley, R. J. 2004, PASP, 116, 597

Gal-Yam, A., et al. 2007, ApJ, 656, 372

Gelman, A., Carlin, J., Stern, H., \& Rubin, D. 1995, Bayesian Data Analysis (Boca Raton: Chapman \& Hall)

Germany, L. M., Reiss, D. J., Schmidt, B. P., Stubbs, C. W., \& Suntzeff, N. B. 2004, A\&A, 415, 863

Grazian, A., et al. 2006, A\&A, 449, 951 
Hillebrandt, W., \& Niemeyer, J. C. 2000, ARA\&A, 38, 191

Jha, S., et al. 2006, AJ, 131, 527

Johnson, B. D., \& Crotts, A. P. S. 2006, AJ, 132, 756

Kaiser, N., et al. 2005, BAAS, 37, 1409

Kashikawa, N., et al. 2004, PASJ, 56, 1011

Knop, R. A., et al. 2003, ApJ, 598, 102

Kuznetsova, N. V., \& Connolly, B. M. 2007, ApJ, 659, 530

Li, W., Filippenko, A. V., Treffers, R. R., Riess, A. G., Hu, J., \& Qiu, Y. 2001, ApJ, 546, 734

Madau, P., della Valle, M., \& Panagia, N. 1998, MNRAS, 297, L17

Mannucci, F., Della Valle, M., \& Panagia, N. 2006, MNRAS, 370, 773

Mannucci, F., Della Valle, M., Panagia, N., Cappellaro, E., Cresci, G., Maiolino, R., Petrosian, A., \& Turatto, M. 2005, A\&A, 433, 807

Maoz, D., \& Gal-Yam, A. 2004, MNRAS, 347, 951

Neill, J. D., et al. 2006, AJ, 132, 1126

Nobili, S., Goobar, A., Knop, R., \& Nugent, P. 2003, A\&A, 404, 901

Nugent, P., Kim, A., \& Perlmutter, S. 2002, PASP, 114, 803

Nugent, P., et al. 2006, ApJ, 645, 841

Perlmutter, S., et al. 1999, ApJ, 517, 565

Poznanski, D., Gal-Yam, A., Maoz, D., Filippenko, A. V., Leonard, D. C., \& Matheson, T. 2002, PASP, 114, 833
Poznanski, D., et al. 2007, MNRAS, submitted (arXiv: 0707.0393)

Rajala, A. M., et al. 2005, PASP, 117, 132

Richardson, D., Branch, D., Casebeer, D., Millard, J., Thomas, R. C., \& Baron, E. 2002, AJ, 123, 745

Riess, A. G., et al. 2004a, ApJ, 607, 665 . 2004b, ApJ, 600, L163

Scannapieco, E., \& Bildsten, L. 2005, ApJ, 629, L85

Schechter, P. 1976, ApJ, 203, 297

Schlegel, D. J., Finkbeiner, D. P., \& Davis, M. 1998, ApJ, 500, 525

Sollerman, J., et al. 2006, in Beyond Einstein-Physics for the 21st Century, ed. A. M. Cruise \& L. Ouwehand (ESA SSP-637; Noordwijk: ESA), 14

Strolger, L.-G., et al. 2004, ApJ, 613, 200

Stubbs, C. W., Sweeney, D., \& Tyson, J. A. 2004, BAAS, 36, 1527

Sullivan, M., et al. 2006a, AJ, 131, 960 2006b, ApJ, 648, 868

Tonry, J. L., et al. 2003, ApJ, 594, 1

Vanden Berk, D. E., et al. 2001, AJ, 122, 549

Wolf, C., Wisotzki, L., Borch, A., Dye, S., Kleinheinrich, M., \& Meisenheimer, K. 2003, A\&A, 408, 499

Wood-Vasey, W. M., et al. 2004, NewA Rev., 48, 637

Woosley, S., \& Janka, T. 2005, Nature Phys., 1, 147 